\title{
A rare case of isolated atrial myocarditis
}

Singapore Med J 2021; 62(11): 610-614 https://doi.org/10.11622/smedj.2021227

Dear Sir,

A 45-year-old Caucasian woman with a history of Type 1 insulin-dependent diabetes mellitus presented with fever, nausea and malaise. She was treated with empirical antibiotics but returned with complaints of dizziness after one week. Physical examination showed a blood pressure of 107/52 $\mathrm{mmHg}$ and signs of mild fluid overload.

Electrocardiography (ECG) showed junctional bradycardia at 33 beats per minute (bpm). Chest radiograph showed increased interstitial shadowing with bilateral pleural effusions, consistent with mild cardiac failure. Serum high-sensitive troponin $\mathrm{T}$ level was mildly elevated at 45.1 (normal range $[\mathrm{NR}] \leq 14) \mathrm{pg} / \mathrm{mL}$ and $\mathrm{N}$-terminal pro-B-type natriuretic peptide (NT-proBNP) level was 1,044 (NR 0-125) $\mathrm{pg} / \mathrm{mL}$. The rest of the laboratory results were as follows: haemoglobin level $11.1 \mathrm{~g} / \mathrm{dL}$, white blood cell count $9.59 \times 10^{9} / \mathrm{L}$ (with mild lymphopenia), platelet count $458 \times 10^{9} / \mathrm{L}$, urea level $8.0 \mathrm{mmol} / \mathrm{L}$, creatinine level $84 \mu \mathrm{mol} / \mathrm{L}$, albumin level $40 \mathrm{~g} / \mathrm{dL}$, aspartate aminotransferase level $284 \mathrm{U} / \mathrm{L}$ and alanine aminotransferase level $167 \mathrm{U} / \mathrm{L}$. Her C-reactive protein level was $31.1 \mathrm{mg} / \mathrm{L}$ and procalcitonin level was less than $0.06 \mathrm{ng} / \mathrm{mL}$.

The patient was initially administered the empiric antiviral Tamiflu (oseltamivir) and antibiotics doxycycline and Augmentin (co-amoxiclav). She was also treated with frusemide for diuresis. Continuous ECG monitoring showed infrequent sinus pauses of up to 8.6 seconds (Fig. 1). Although the patient felt fleeting, mild dizziness, no syncope or haemodynamic instability was observed. Temporary transvenous pacing was proposed, but a conservative approach of careful monitoring in the high-dependency unit was adopted owing to the patient's refusal to undergo the procedure.

Echocardiography revealed a mildly dilated left atrium with diffusely thickened left atrial wall measuring 6 mm (Fig. 2). Other chamber walls and sizes were normal. Left ventricular systolic function was normal, with no regional wall motion abnormalities detected. There was a small circumferential pericardial effusion. Estimated pulmonary artery systolic pressure was elevated at $61 \mathrm{mmHg}$. Computed tomography (CT) of the chest confirmed diffuse thickening of the left atrium and left atrial appendage, with bilateral pleural effusions (Fig. 3). No mediastinal or hilar lymphadenopathy was noted. Respiratory virus panel polymerase chain reaction test was negative. Autoimmune markers were also normal.

Fluorodeoxyglucose (FDG) positron emission tomography (PET)-CT imaging for FDG avid foci showed heterogenous uptake along the wall of the left atrium, including the left atrial appendage (Fig. 4). There was no involvement of the right atrium or both ventricles. This was indicative of an ongoing inflammatory process localised to the left atrium.

These findings were consistent with the diagnosis of isolated left atrial myocarditis. The patient was advised to undergo a biopsy of her atrial tissue for histological diagnosis before commencing immunosuppressive therapy; however, she declined. She was started on high-dose oral prednisolone at $1 \mathrm{mg} / \mathrm{kg} /$ day on Day 9 of hospital admission. The sinus pauses completely resolved, but she developed atrial fibrillation and atrial flutter with rapid ventricular conduction between $100 \mathrm{bpm}$ and 150 bpm, requiring nebivolol for rate control. No significant ventricular arrhythmias were noted throughout the admission. After 18 days of atrial fibrillation and flutter, the patient spontaneously reverted to sinus rhythm. Troponin levels normalised after starting oral steroids. Follow-up cardiac magnetic resonance (MR) imaging prior to discharge showed mild late gadolinium contrast enhancement (LGE) in parts of the left atrial wall measuring 4-5 mm in thickness, indicative of patchy inflammation or fibrosis in the left atrium. No inflammation or fibrosis was noted in the right atrium or ventricular walls.

The patient experienced infrequent episodes of short-lasting palpitations after discharge, with no further episodes of fever or dizziness. Serial 24-hour ambulatory ECG monitoring showed short runs of non-sustained atrial tachycardia, which reduced in frequency over time. Repeat echocardiography also showed resolution of the left atrial wall thickening and pericardial effusion (Fig. 2b). The pulmonary artery pressure also normalised. The patient was gradually weaned off prednisolone over six months. Her serum troponin levels remained normal throughout follow-up. NT-proBNP levels were $408 \mathrm{pg} / \mathrm{mL}$ and $164 \mathrm{pg} / \mathrm{mL}$ at one month and

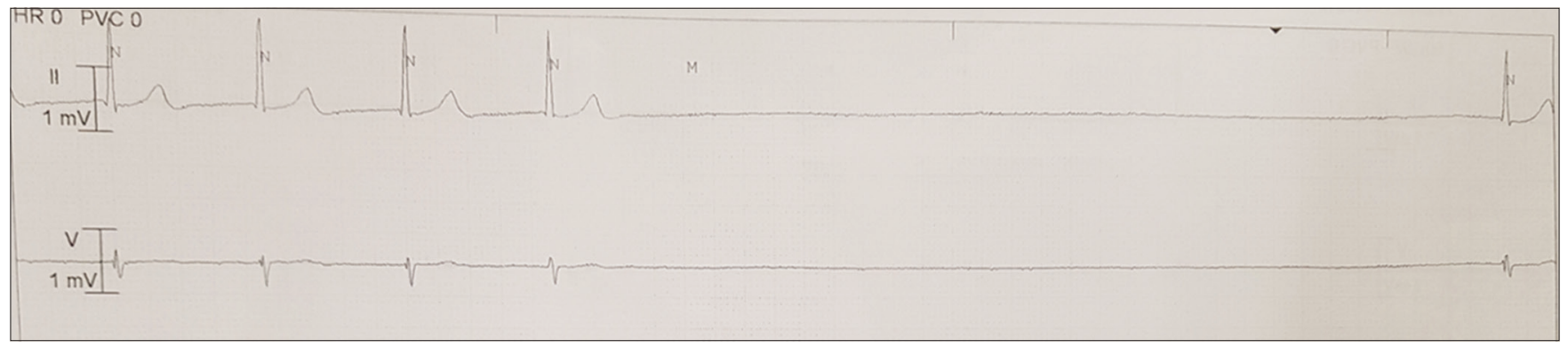

Fig. 1 Continuous ECG monitoring strip shows one episode of sinus pause. The longest pause detected in our patient was 8.6 seconds. 

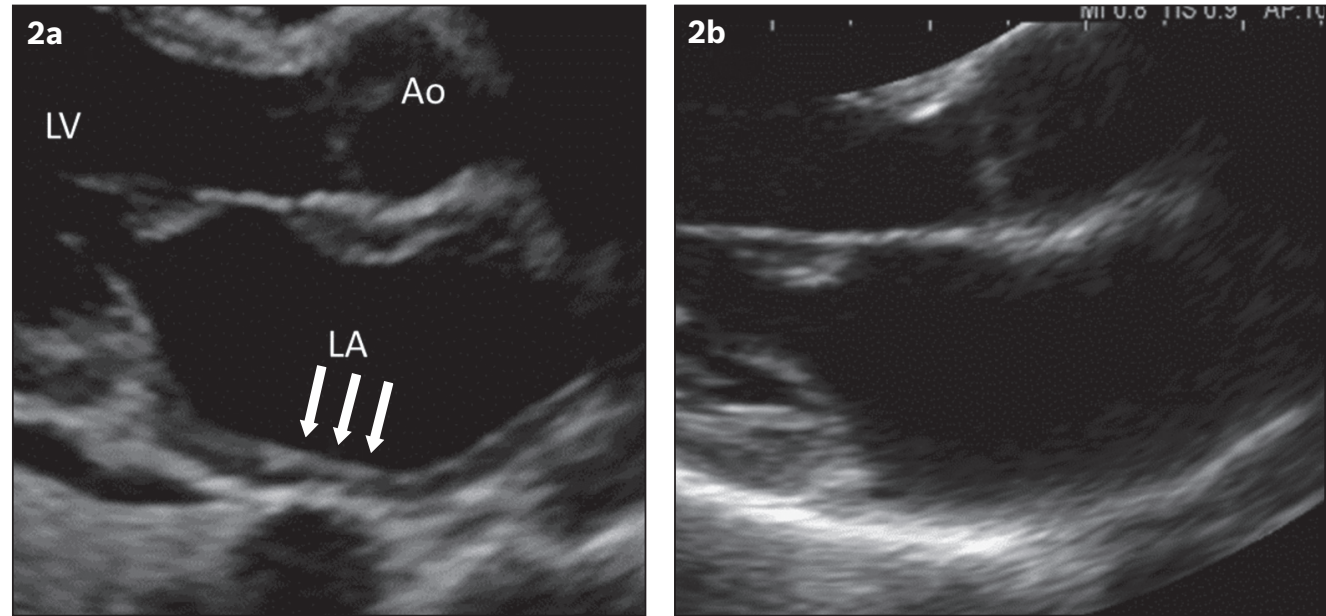

Fig. 2 Echocardiogram shows a left atrium (LA) wall that was (a) diffusedly thickened (arrows) before corticosteroids and (b) of normal thickness six months after treatment. The left ventricle (LV) wall was of normal thickness at initial presentation. Ao: aorta
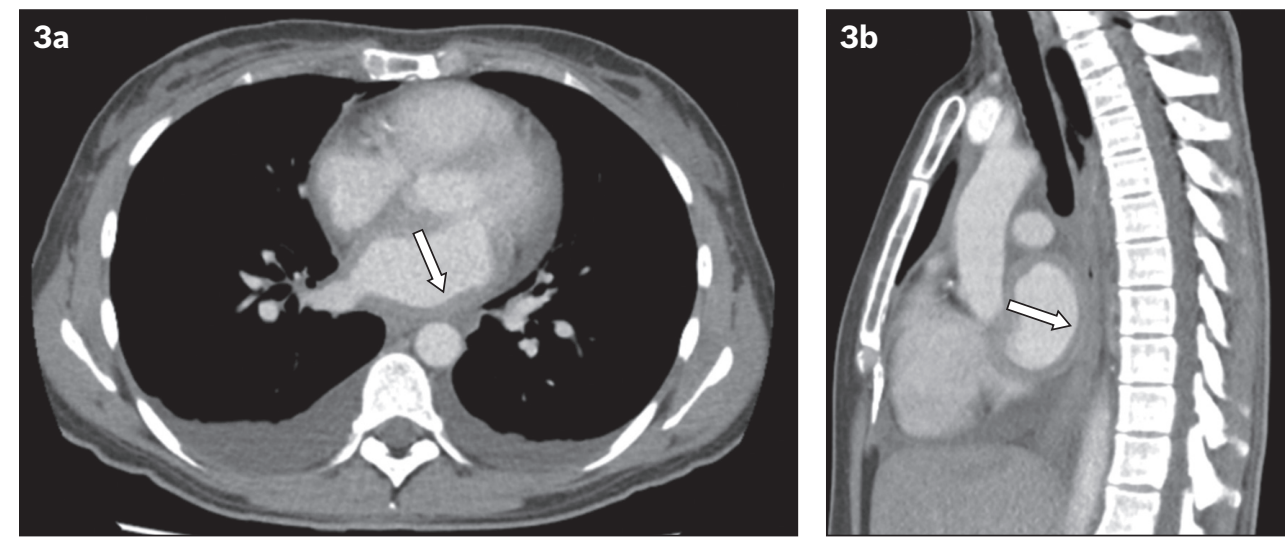

Fig. 3 CT images of the chest in (a) transverse view and (b) sagittal view show diffused thickening of the left atrium (arrows) and bilateral pleural effusions.

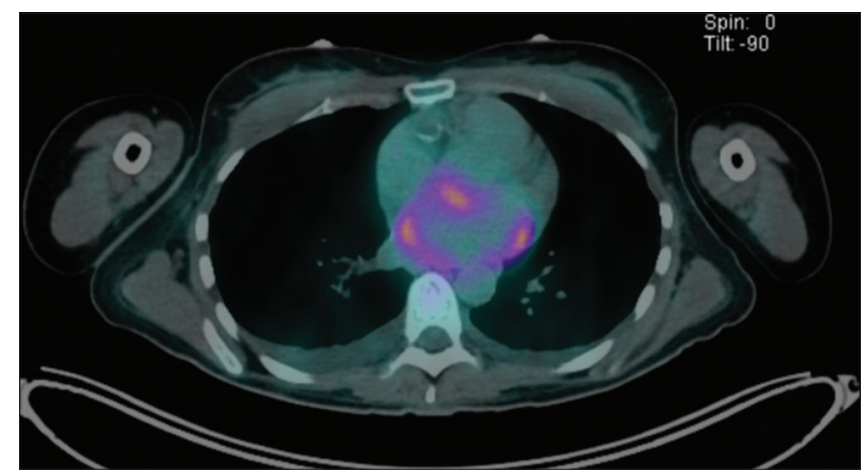

Fig. 4 PET (positron emission tomography)-CT image shows heterogenous fluorodeoxyglucose uptake along the wall of the left atrium, including the left atrial appendage.

three months after discharge, respectively. These levels completely normalised to $100 \mathrm{pg} / \mathrm{mL}$ after six months. One year after initial presentation, the patient remained asymptomatic and in sinus rhythm.

Atrial involvement can occur concurrently with ventricular myocarditis in patients with myocarditis of different aetiologies. ${ }^{(1)}$ However, isolated atrial myocarditis is an uncommon presentation of acute myocarditis. The most commonly reported aetiology of isolated atrial myocarditis is giant cell myocarditis. ${ }^{(2)}$ Other reported causes include sarcoidosis and lymphocytic myocarditis. ${ }^{(3)}$ The clinical features and outcomes of previously reported cases of atrial myocarditis are summarised in Table I. The aetiology in our patient was uncertain, as she had declined a biopsy. We had presumed it to be an inflammatory cause of either sarcoidosis or giant cell myocarditis, and treated her with high-dose corticosteroid therapy, with gradual weaning over six months.

Most patients with isolated atrial myocarditis present with non-specific symptoms such as fever, breathlessness and mild fluid overload, or are incidentally diagnosed when they undergo atrial fibrillation ablation or cardiac surgery, or on postmortem. ${ }^{(2,4)}$ Atrial 

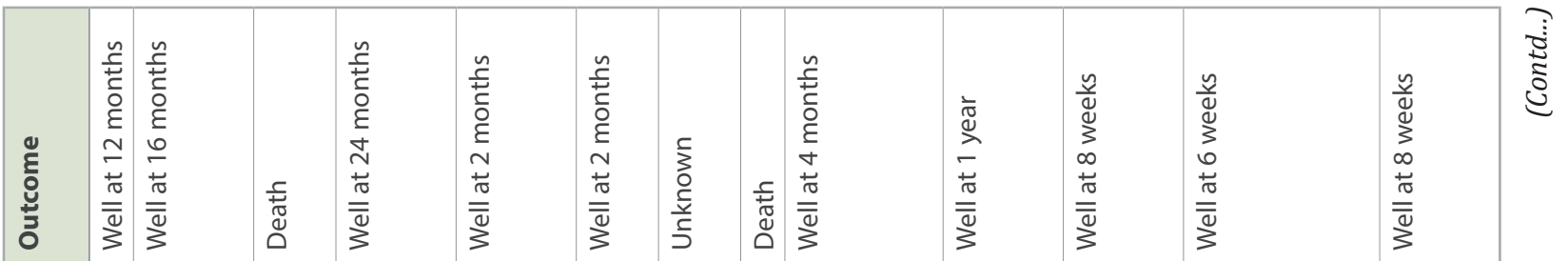

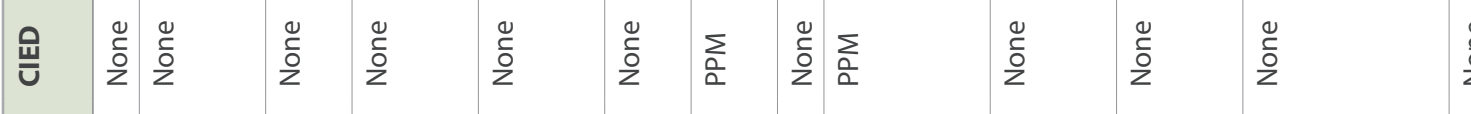

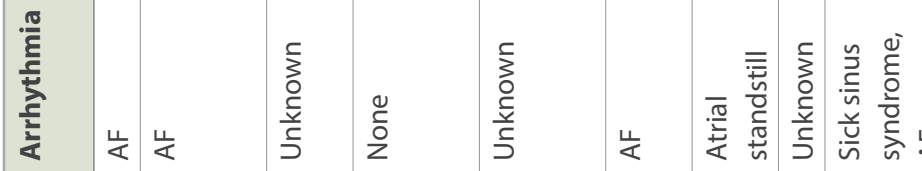

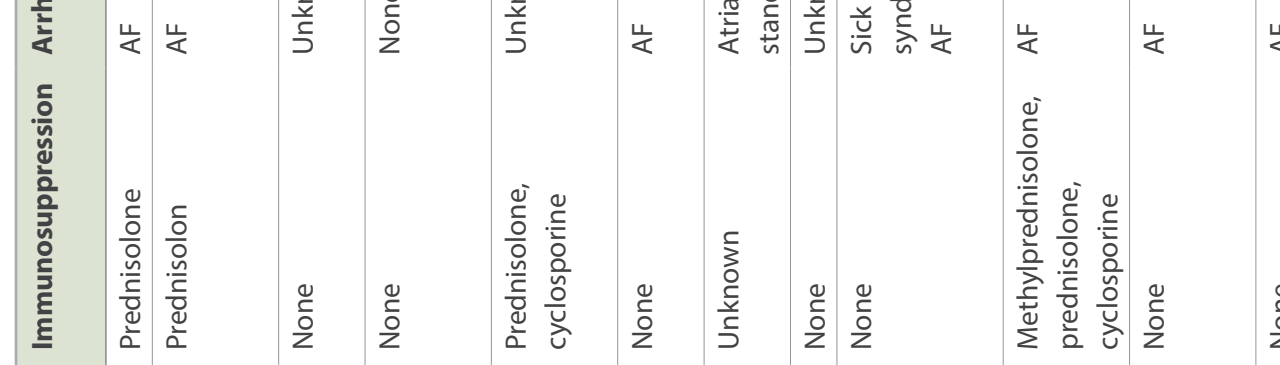

\section{$\frac{5}{\frac{10}{7}}$}

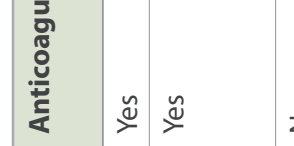

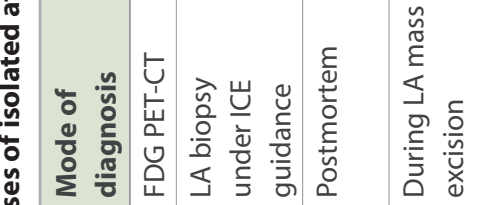

旁言

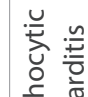

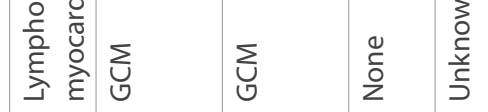

동홍

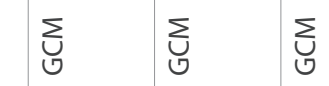

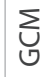

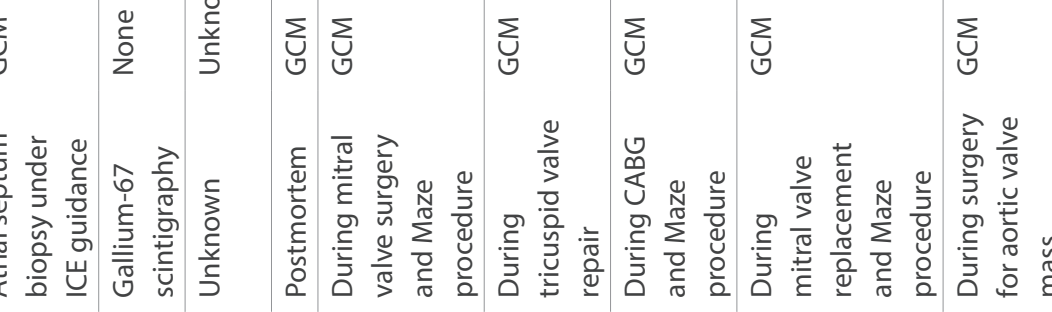

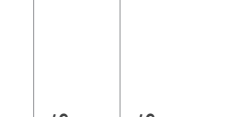

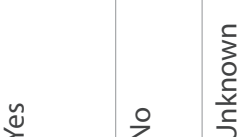

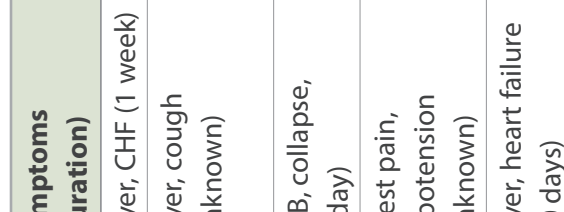

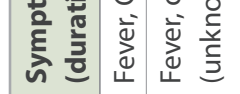

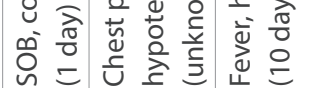

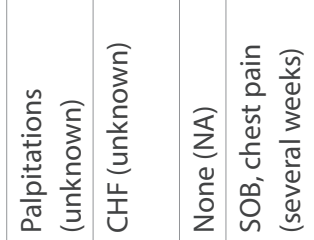

离

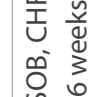

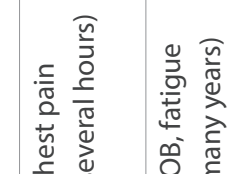

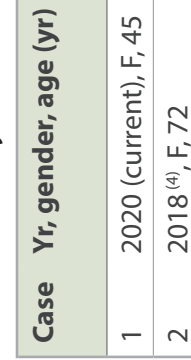

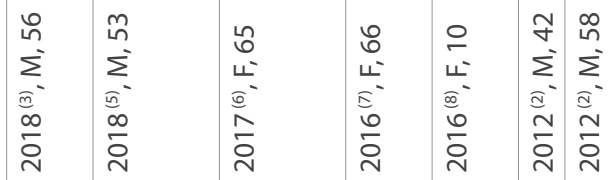

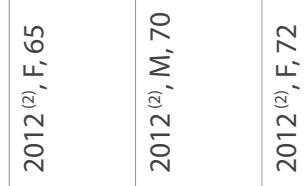

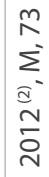

$\frac{0}{2}$

$\stackrel{\varrho}{\rightleftharpoons}$

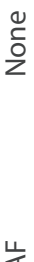

岩 


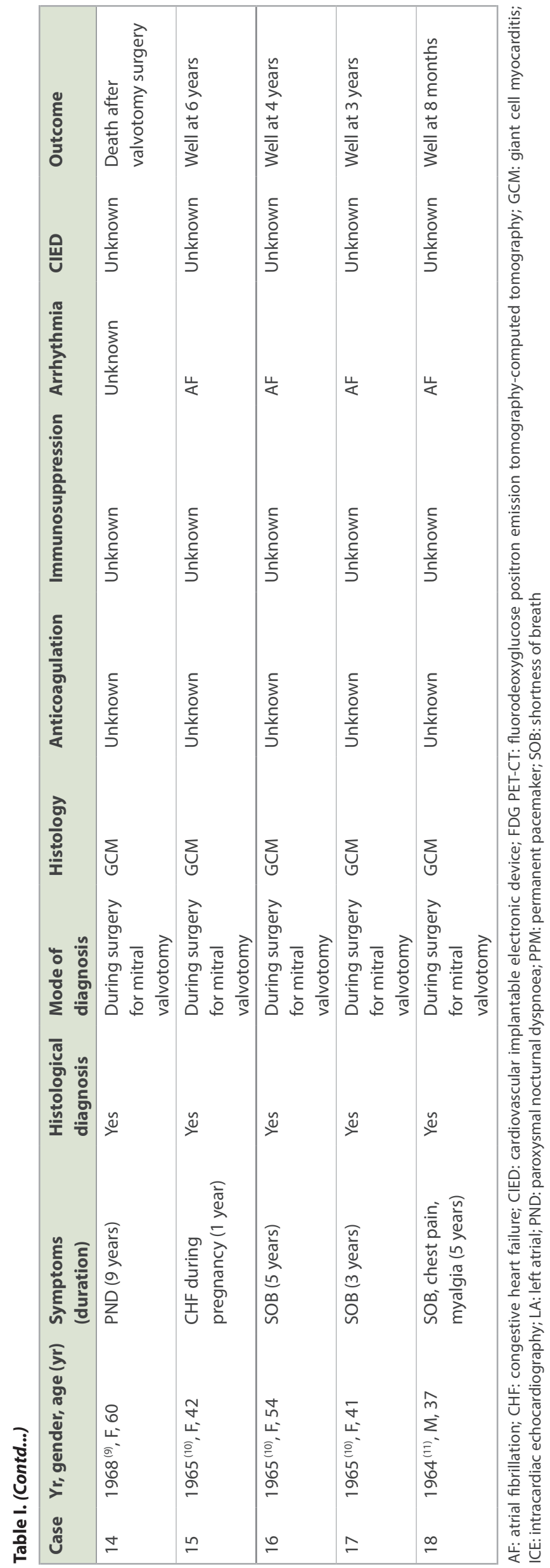

myocarditis is usually associated with atrial arrhythmias, most commonly atrial fibrillation. ${ }^{(2)}$ Atrial dysfunction increases the risk of thrombosis and cerebrovascular embolic events, which may be mitigated by anticoagulation. In some cases, persistent atrial standstill can also occur, requiring permanent pacemaker implantation. ${ }^{(8)}$ Our patient was found to have significant sinus pauses with infrequent episodes of fleeting dizziness during admission. In acute management of sinus node dysfunction, temporary pacing is indicated in patients with severe symptoms and hemodynamic compromise. ${ }^{(12)}$ However, the patient declined temporary insertion of transvenous pacemaker. Eventually, the sinus pauses resolved, converted to atrial fibrillation and flutter, and subsequently reverted to sinus rhythm following steroid therapy.

A definitive diagnosis requires histological confirmation via an atrial wall biopsy. Histological diagnosis is also important to guide the choice and duration of immunosuppression. Endomyocardial biopsy typically involves biopsy of the interventricular septum from the right ventricle via a transjugular venous approach. In our patient, this would require biopsy of the thinner interatrial septum under transoesophageal or intracardiac echocardiography guidance, a higher-risk procedure, which the patient declined. An alternative approach would be via surgical resection of the left atrial appendage.

Diagnosis of atrial myocarditis using transoesophageal echocardiography, gallium-67 scintigraphy, FDG PET-CT or cardiac MR imaging has been described..$^{(2,6,7)}$ In our patient, a markedly thickened left atrial wall could be observed on echocardiography early in the disease. This finding corroborated with subsequent chest CT, FDG PET-CT and cardiac MR imaging. Cardiac MR imaging and FDG PET-CT have complementary roles in the assessment of suspected myocardial inflammatory disease. We opted for FDG PET-CT as the initial imaging for diagnosis, assessment of overall disease involvement and localising extracardiac involvement that was amenable to biopsy. Unfortunately, the FDG PET-CT only revealed isolated increased left atrial wall FDG uptake, without any conclusive evidence of extracardiac involvement. No abnormal FDG uptake was observed at the right atrial or both ventricular walls. Subsequently, cardiac MR imaging was performed 20 days after FDG PET-CT, which showed patchy LGE at the left atrial wall without any definite abnormality in the right atrium, interventricular septum, right ventricle or left ventricle. Both FDG PET-CT and cardiac MR imaging revealed an isolated left atrial abnormality, which was related to an inflammatory process. The manifestation of sinus pauses indicated the involvement of the right atrium, which is not uncommon in previously reported cases. However, the degree of inflammation and extent of involvement of the right atrium may have been too subtle to be detected on both imaging modalities in our patient.

Immunosuppressive therapy in patients presenting with viral myocarditis remains controversial and is, therefore, not routinely used. ${ }^{(13)}$ Combination immunosuppression with drugs such as cyclosporine or azathioprine is, however, recommended if the underlying aetiology is giant cell myocarditis. ${ }^{(14)}$ Nevertheless, the duration of immunosuppressant therapy for isolated atrial 
myocarditis is presently unclear. The prognosis of atrial giant cell myocarditis appears to be much better than that observed in ventricular giant cell myocarditis, while the natural course and pathogenesis also seem to be substantially different. ${ }^{(2)}$ In the Mayo Clinic case series of atrial giant cell myocarditis, only one out of six patients received long-term immunosuppression. In our patient, who had Type 1 diabetes mellitus, a high dose of a potent corticosteroid such as methylprednisolone would also risk derangement of her blood sugar control, thereby increasing the risk of secondary bacterial infection. We were also concerned about the risks of starting combination immunosuppression for her without histological confirmation. Finally, in view of the cardiac rhythm disturbance and atrial involvement, we successfully treated our patient by starting with a pulse of oral prednisolone on Day 9 of admission, with gradual tapering over six months.

There is no recommended method of monitoring response to therapy. The serum troponin level of our patient was only mildly elevated on initial presentation and normalised quickly with steroid therapy. NT-proBNP appeared to be a useful biomarker to track her response over time. BNP is produced mainly by the ventricles but also, to a smaller degree, from the atria. NT-proBNP level was likely elevated owing to the atrial inflammation. It could also be attributable to stiff left atrial syndrome resulting from reduced left atrial compliance, pulmonary venous hypertension and manifestation of fluid overload. ${ }^{(15)}$

In summary, isolated atrial myocarditis is an uncommon presentation of acute myocarditis and is associated with supraventricular tachyarrhythmias and bradyarrhythmias. Based on the limited available literature, its clinical course and prognosis also appear different and possibly more favourable than those of ventricular myocarditis. In the absence of definitive histological diagnosis, imaging with echocardiography, FDG PET-CT or cardiac MR imaging may be useful to aid diagnosis. Our patient responded well to steroid therapy, and we report a good short-to-intermediate-term prognosis. The benefits of long-term immunosuppressive therapy remain unclear and warrant further investigation.

Yours sincerely,

Choon Pin $\underline{\mathrm{Lim}}^{1}$, Paul Toon Lim $\underline{\mathrm{Chiam}}^{2}$, Hee Kit $\underline{\mathrm{Lai}}^{3}$, Yin Ling $\underline{\mathrm{Koh}}^{4}$

${ }^{1}$ The Heart and Vascular Centre, Mount Elizabeth Novena Hospital, ${ }^{2}$ The Heart and Vascular Centre, Mount Elizabeth Hospital, ${ }^{3}$ Radiology Department, Mount Elizabeth Novena Hospital, ${ }^{4}$ The Novena Medical Specialists, Mount Elizabeth Novena Hospital, Singapore. limchoonpin@gmail.com

\section{References}

1. Begieneman MP, Emmens RW, Rijvers L, et al. Ventricular myocarditis coincides with atrial myocarditis in patients. Cardiovasc Pathol 2016; 25:141-8.

2. Larsen BT, Maleszewski JJ, Edwards WD, et al. Atrial giant cell myocarditis: a distinctive clinicopathologic entity. Circulation 2013; 127:39-47.

3. Tse R, Garland J, Kesha K, et al. A rare case of isolated atrial myocarditis causing death with no post mortem computed tomography scan correlation. Am J Forensic Med Pathol 2018; 39:123-5.

4. Kumagai K, Shirakura T, Minami K, Oshima S. Atrial giant cell myocarditis after atrial fibrillation ablation. Eur Heart J Case Rep 2018; 2:yty065.

5. Tanyeli O, Dereli Y, Gormus N, Avunduk MC. Left atrial giant cell myocarditis presenting as a tumor: first-in-man case report. Braz J Cardiovasc Surg 2018; 33:306-8.

6. Arai H, Kuroda S, Yoshioka K, Mizukami A, Matsumura A. Images of atrial giant cell myocarditis. Eur Heart J Cardiovasc Imaging 2018 ; $19: 243$.

7. Kotani K, Kawabe J, Higashiyama S, Yoshida A, Shiomi S. Diffuse gallium-67 accumulation in the left atrial wall detected using SPECT/CT fusion images. Case Rep Radiol 2016; 2016:6374584.

8. Prabhu MA, Srinivas Prasad BV, Thajudeen A, Namboodiri N. Persistent atrial standstill in acute myocarditis. Indian Pediatr 2016; 53:162-4.

9. Gillie I, Fox H. Mitral stenosis together with a giant cell myocarditis limited to the left atrium. J Clin Pathol 1968; 21:750-2.

10. Husband EM, Lannigan R. Unusual giant cell lesions in biopsy specimen of left atrial appendages in mitral stenosis. Br Heart J 1965; 27:269-73.

11. McCrea PC, Childers RW. Two unusual cases of giant cell myocarditis associated with mitral stenosis and with Wegener's syndrome. Br Heart J 1964; $26: 490-8$.

12. Kusumoto FM, Schoenfeld MH, Barrett C, et al. 2018 ACC/AHA/HRS guideline on the evaluation and management of patients with bradycardia and cardiac conduction delay: executive summary: a report of the American College of Cardiology/American Heart Association Task Force on clinical practice guidelines, and the Heart Rhythm Society. J Am Coll Cardiol 2019; 74:932-87.

13. Mason JW, O'Connell JB, Herskowitz A, et al. A clinical trial of immunosuppressive therapy for myocarditis. The Myocarditis Treatment Trial Investigators. N Engl J Med 1995; 333:269-75.

14. Kandolin R, Lehtonen J, Salmenkivi K, et al. Diagnosis, treatment, and outcome of giant-cell myocarditis in the era of combined immunosuppression. Circ Heart Fail $2013 ; 6: 15-22$.

15. Mehta S, Charbonneau F, Fitchett DH, et al. The clinical consequences of a stiff left atrium. Am Heart J 1991; 122(4 Pt 1):1184-91. 